\title{
PARTICLE DYNAMICS IN THE RMP ERGODIC LAYER UNDER THE INFLUENCE OF EDGE PLASMA TURBULENCE
}

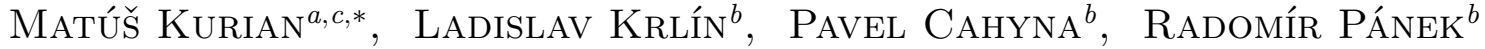 \\ ${ }^{a}$ Faculty of Mathematics and Physics, Charles University \\ ${ }^{b}$ Institute of Plasma Physics AS CR, v.v.i. \\ c Gymnázium Jura Hronca, Bratislava \\ * corresponding author: kurian@gjh.sk
}

\begin{abstract}
The complex processes in edge tokamak plasma are affected (among others) both by resonant magnetic perturbation (RMP) and by plasma turbulence. RMP is nowadays considered to be a candidate for the mitigation of the edge-localized modes (ELMs). The effect of plasma turbulence in the edge region has not been studied yet. Since both RMP and plasma turbulence should influence plasma dynamics, studies of their simultaneous effect have potential practical implications. Using a simplified model of the turbulence and single-particle simulations, we discovered that its effect at realistic amplitudes changes the ion dynamics significantly. We expect that the turbulence has a similar effect on electrons, thus potentially influencing the ELM mitigation mechanism.
\end{abstract}

KEYWORDS: resonant-magnetic perturbation, plasma turbulence, non-linear dynamics.

\section{INTRODUCTION}

It has been observed that RMP (Resonant Magnetic Perturbation), created by auxiliary coils, can mitigate the ELM instability on several tokamaks, including the DIII-D [1]. Earlier explanations state that the stochasticity of the magnetic field lines enables fast escape of electrons, which results in a change of the density gradient. Experiments cast doubt upon this simple explanation, and as yet no convincing explanation of the mitigation of ELM has been found. This calls for a discussion of the roles of other mechanisms that can appear in this region, and also of the effect of all sorts of particles involved in the process. The generation of a radial electric field in the ergodic layer seems to play an important role.

The usual approach to the generation of a radial electric field considers quiet plasma without any chaotic perturbation of the density of the plasma in this region. However, RMP will surely work in a plasma with some level of electrostatic turbulence, which seems to be a generic phenomenon of an edge tokamak plasma.

This turbulence causes the generation of blobs made of potential hills and valleys in the potential landscape [2]. It has been observed that the dynamics of electrons and ions is different in such potential. While electrons tend to be trapped in its structures, ions may also be trapped, or - depending on their mass and energy - they may be subject to anomalous diffusion [3]. It was proposed in 4 that such differences can result in the generation of an electrostatic field.

Furthermore, considering the effect of the ergodic layer, plasma particles will be influenced not only by the electrostatic turbulence, but also by the toroidal magnetic field and the magnetic field of overlapping magnetic islands. Thus there are two potential mech- anisms for generating of a radial electric field in the system.

However, a self-consistent simulation of the concurrent effect of the ergodic layer and the electrostatic turbulence on plasma particles presents an extremely difficult problem that has not been yet solved. Perhaps the first important step towards a solution appears in [5. There, the influence of RMP on electrostatic turbulence is discussed in the framework of fluid dynamics and the extended Hasegawa-Wakatani model. In our opinion, the effect on all types of particles also needs to be investigated.

The first step can be formulated as follows: Under which conditions will the effect of electrostatic turbulence overshadow (or, at least be comparable with) the effect of the ergodic layer of RMP? To answer this basic question, it is necessary to study the particle dynamics in the complicated system of electrostatic turbulence potential and in the ergodic layer. The complications center around the turbulent electrostatic potential, the form of which has to be estimated.

A full, self-consistent solution can therefore be expected only in a theoretical approach. Nevertheless, an approach employing a simple model of the turbulence can provide us at least a glimpse of what happens in this complicated region.

\section{MODEL}

We start with a discussion of the effect of low frequency electrostatic turbulent potential perturbation, which can compete with the effect of a system of stochastic field lines. This turbulence is the result of the instability of resistive drift waves (e.g. in 2]). We modelled the potential by a time-stationary and spatially periodical function, which we had used in $[3,4$. The time 


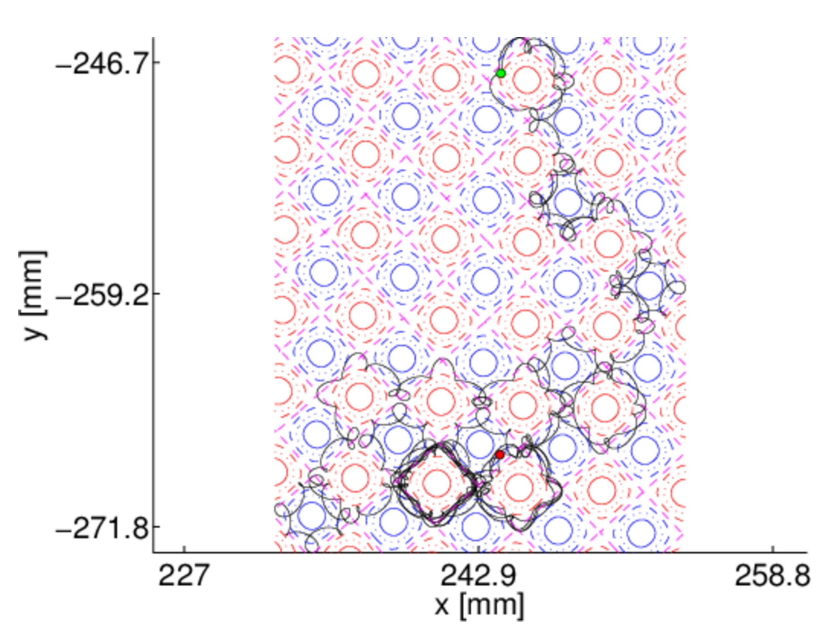

(a) C, $U=20 \mathrm{~V}$, not trapped

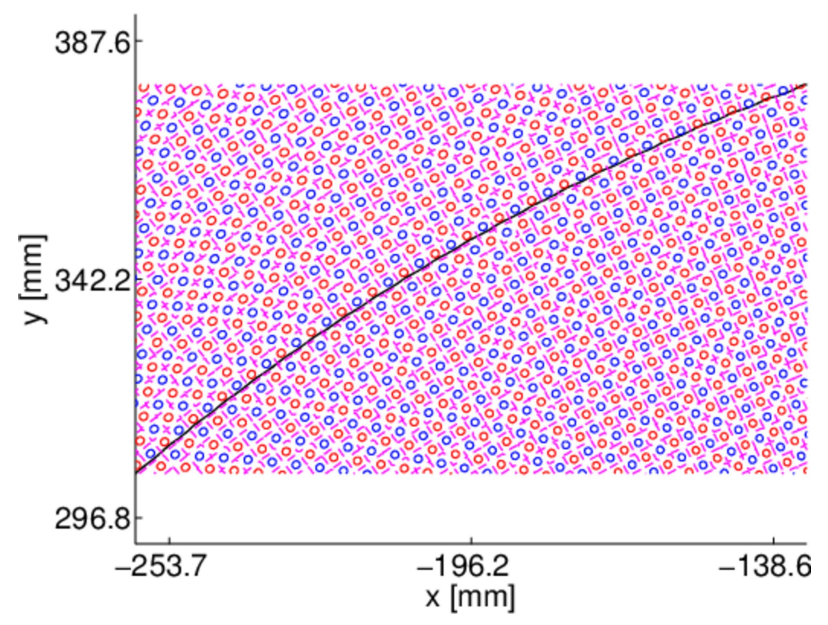

(c) e, $U=20 \mathrm{~V}$, not trapped

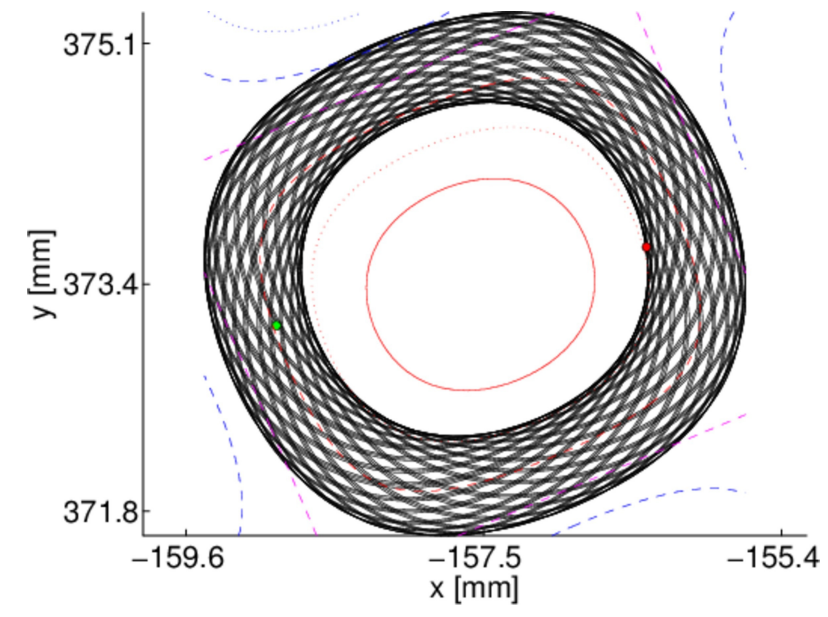

(b) C, $U=20 \mathrm{~V}$, trapped

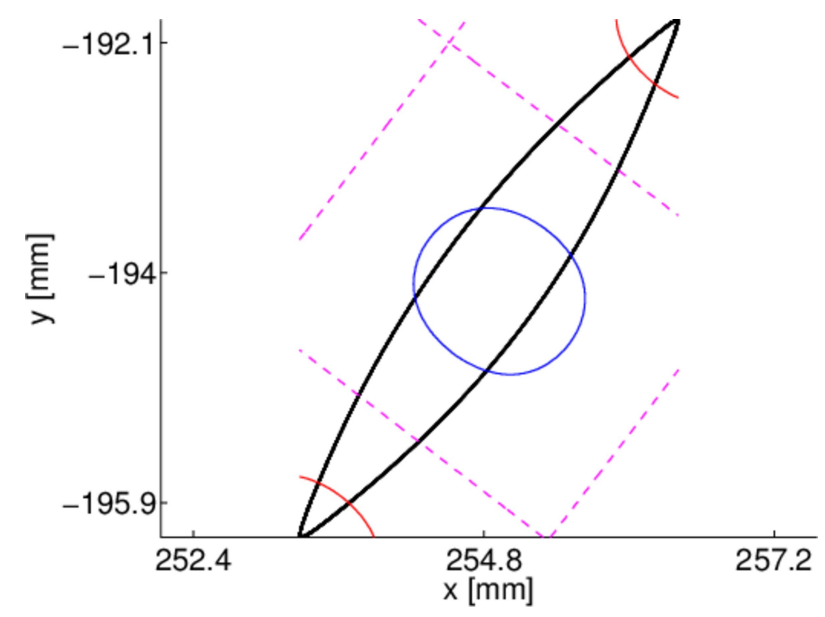

(d) e, $U=20 \mathrm{~V}$, trapped

FigURE 1. Comparison of the trajectories of carbon ions and electrons - projection: a trapped trajectory, as well as a non-trapped trajectory, is presented in each case. The trajectories in the non-trapped case are also different: the electron tends to follow a path parallel to the separatrix (see Fig. 1c), whereas the ion demonstrates a fairly complicated random walk (see Fig. 1a).

stationarity is justified by well-based experimental evidence (recently in [5]) of the long correlation of potential perturbation along magnetic field lines. The spatial periodicity models the appearance of "hills" and "blobs" in the electron density distribution. In the literature, this potential is called "egg-crate potential" — see an analogy in solid state physics [6]. Obviously, both assumptions - the time independence and the spatial periodicity of blobs - represent strong approximations. However, they enable us to obtain some results, which can later be verified on more realistic models.

We describe the particle motion by means of a singleparticle Hamiltonian, which includes the toroidal and poloidal magnetic fields, the resonant magnetic perturbation, and a corresponding electrostatic perturbation, respecting the helical character of the field lines. The presence of two rows of magnetic islands of RMP is included as a perturbation of the main tokamak field. The electrostatic potential follows the helicity, given by the safety factor $q_{\mathrm{f}}$. To avoid the effect of drifts in curvilinear field lines in toroidal representation, and the generation of an additional sort of chaos resulting from such geometry, we limit our procedure to a cylindrical system with periodic boundary conditions. The final form of the Hamiltonian is (using [7, 8])

$$
\begin{gathered}
\mathcal{H}=\frac{1}{2 M}\left[p_{r}^{2}+\frac{1}{r^{2}}\left(p_{\theta}-q A_{\theta}\right)^{2}+\left(p_{z}-q A_{z}\right)^{2}\right] \\
+U_{0} q \cos (k r) \cos \left[k r_{\mathrm{d}}\left(\theta-\frac{z}{R_{0} q_{\mathrm{f}}}\right)\right]
\end{gathered}
$$

where $A_{\theta}=\psi=\frac{1}{2} B_{0} r^{2}$

$$
\text { and } \begin{aligned}
A_{z}=-\frac{1}{R_{0}} \int q_{\mathrm{f}}^{-1} \mathrm{~d} \psi-\frac{L \psi}{R_{0}}[ & {\left[\cos \left(2 \theta-z / R_{0}\right)\right.} \\
+ & \left.\cos \left(3 \theta-z / R_{0}\right)\right] \text {. }
\end{aligned}
$$

\section{Method}

We follow the dynamics of particles separately for electrons, protons, and heavier ions. The canonical equations corresponding to the Hamiltonian (1) were 


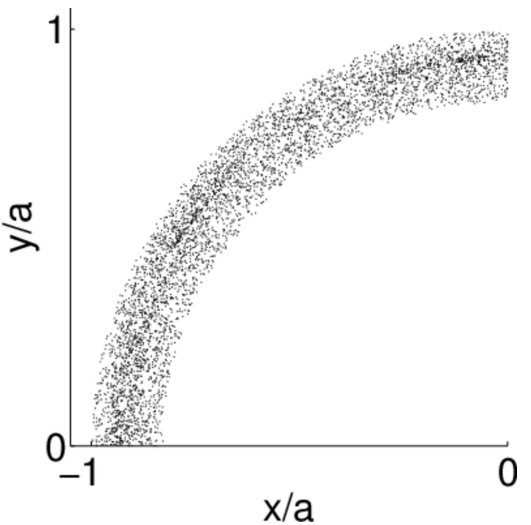

(a) $U=0 \mathrm{~V}$

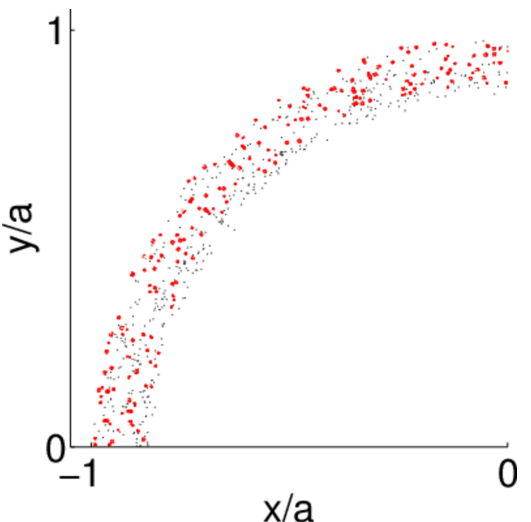

(d) $U=5 \mathrm{~V}$

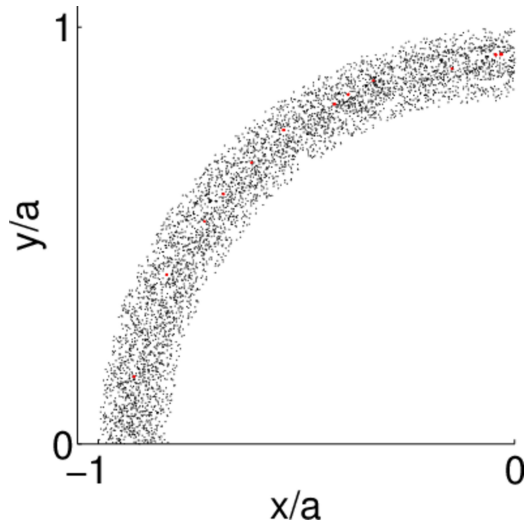

(b) $U=0.1 \mathrm{~V}$

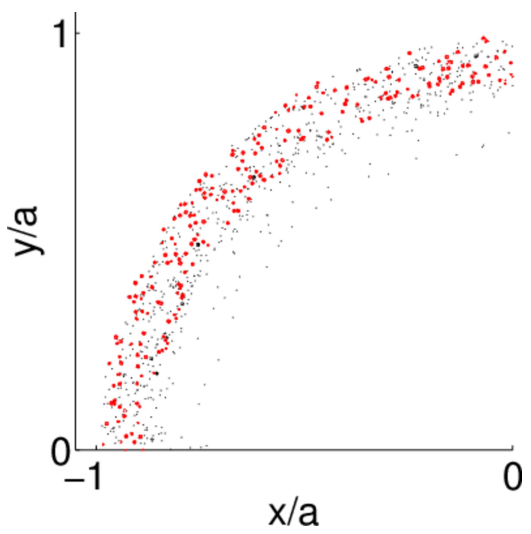

(e) $U=20 \mathrm{~V}$

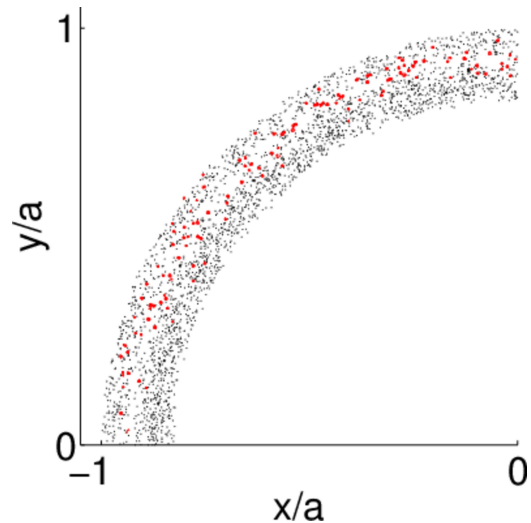

(c) $U=0.5 \mathrm{~V}$

FiguRE 2. A close-up of the surface of section of lithium ions ${ }_{6}^{3} \mathrm{Li}^{+}$: confined trajectories are marked in red, unconfined trajectories in black. It appears that the frequency of the confined trajectories increases with $U_{0}$ : there are no confined trajectories for $U_{0}=0 \mathrm{~V}$ (see Fig. 2a), and their number increases slowly with non-zero $U_{0}$ (Figs. 2b 2d). For amplitudes of $5 \mathrm{~V}$ and above (Figs. 2d, 2e), a majority of the trajectories get confined. Overall 1000 trajectories were run, and there are approximately 15000-20000 points in each surface of section. Parameter $L$, governing the ergodic layer, was set to 0.0007 .

solved by means of the Adams (predictor-corrector) method for particles of different mass at $20 \mathrm{eV}$. Apart from the energy, which is kept constant, three other parameters are to be considered: the charge-mass ratio, $L$, which modulates the ergodic layer, and $U_{0}$, which modulates the electrostatic perturbation. Given that we are interested in behaviour of the most relevant particles of the system - electrons and protons (as well as heavier ions) - the choice of the masses and charges is straightforward, and we are left with a 2-dimensional parametric space $\left(L, U_{0}\right)$.

The first glimpse into the system is provided by a study of the individual trajectories. Although the information gained is qualitative rather than quantitative, it often provides a powerful demonstration. Calculating the dynamics of a batch of electrons over time intervals of $50-100 \mu \mathrm{s}$ is a very challenging numerical task. Hence, we follow the individual trajectories and draw our conclusions from the prevailing trends.

When dealing with a potentially chaotic system, Poincaré sections (surfaces of section) and diffusion are often employed. In our case, the surface of section is the plane $z=\pi R_{0}$ and its periodic extensions.
We mark the intersections of trajectories with the surface of section over a period of approximately 20 revolutions. We consider the case $L, U_{0}=0$ to be the "ground state", and visually compare its Poincaré section with the Poincare sections corresponding to non-zero values of $U_{0}$. In this way we will be able to establish the effect of the electrostatic perturbation. Since this procedure can be performed for particles of various mass, it is possible to compare the effect of electrostatic perturbation on heavy and light particles. Later the amplitude of the stochastic magnetic field $L$ can be changed, while $U_{0}$ is kept constant to determine the influence of the ergodic layer.

It should be noted that the (average) computation time for particles of different mass (and hence the physical time) differs greatly, as it is roughly the distance travelled in the z-direction that is kept constant. Due to the different particles that are used, the physical time of the evolution may exceed the stationary lifespan of the electrostatic perturbation. However, the method provides a long-term insight into the system, which would otherwise be impossible.

An important feature of the method is that it iden- 


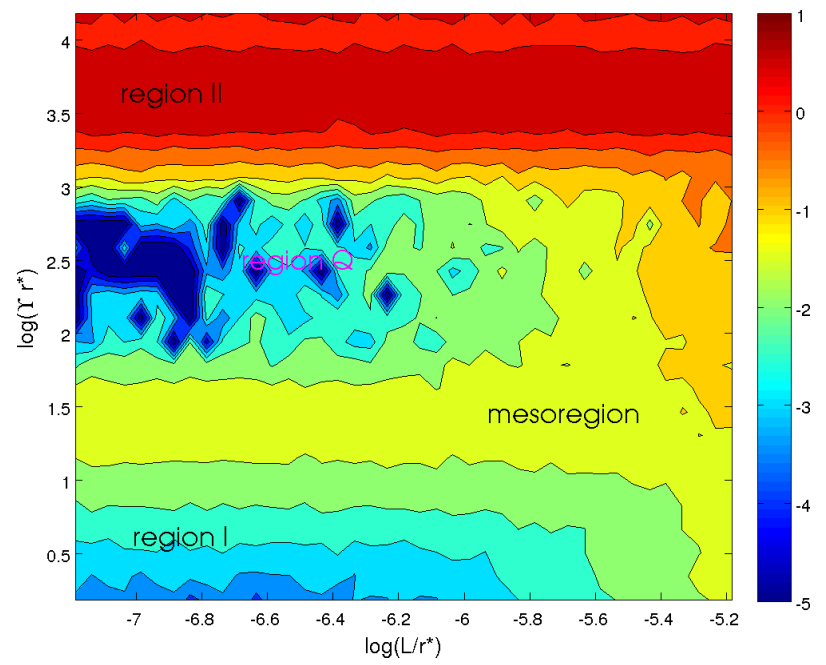

(a) ions

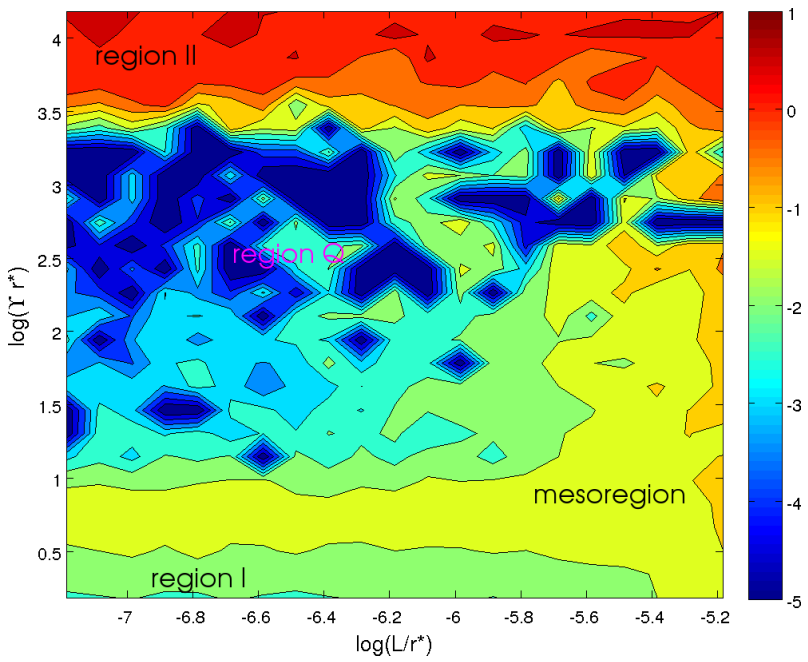

(b) protons

Figure 3. Figure showing the logarithm of the mean radial diffusion coefficient $\alpha$ for carbon ions (Fig. 3a) and for protons (Fig. 3b) as a function of parameters $\Upsilon r^{*}$ and $L / r^{*}$. A total of 250 trajectories were run; $\alpha$ is defined as the slope of the dependence $\overline{[r(t)-r(0)]^{2}}$. On the whole, the system shows that both an increase in $L$ and an increase in $\Upsilon$ generally lead to increased diffusion. Further, for small values of $\Upsilon$ (mainly in region I) $L$ is as important for an increase in diffusion as $\Upsilon$. This does not seem to be the case in region II, where the diffusion is determined mainly by $\Upsilon$. The mesoregion is very interesting: first, $L$ is of much importance in volatile region $Q$, where relatively small changes in $L$ result in transitions from a diffusive state to a non-diffusive state, and vice versa. The region expands with the decrease in particle mass.

tifies the trajectories of the trapped particles. These are the trajectories whose projection onto the surface of section forms a set with a very small diameter. This is consistent with the notion of confinement of the particle in the $(r, \theta)$ plane.

In addition to the Poincaré sections, we study the diffusion in the system. Given that radial diffusion is by far the most relevant in the system, we focus on the radial diffusion coefficient. This was calculated for a bundle of 200 trajectories run over $50 \mu \mathrm{s}$, which is the estimated lifetime of the perturbation. The coefficient was calculated as the slope of the mean square displacement: $D=\overline{[r(t)-r(0)]^{2}}$. We also attempted to identify different diffusion regimes based on the time evolution of the square displacement, namely: normal, hyper, strange, and none.

In order to study the scaling of the diffusion coefficient with respect to particle mass we replace $L, U_{0}$ by $L / r^{*}, \Upsilon r^{*}$, where $\Upsilon \equiv U_{0} / E$, and $r^{*} \equiv r_{\text {gyr }, 0} / a$. This choice is essentially phenomenological, albeit motivated by ideas about relevant dimensionless parameters of the system; it also appears to be partly justified by the results.

\section{Results}

\subsection{Trajectories}

Particle trajectories were first computed in the inertial system. However, drawing an entire 3-D trajectory on a plane is impractical, so we intend to make use of a projection into the $(r, \theta)$ plane. Still, we wish to study the trajectories with respect to the potential structure, which is also $z$-dependent. Fortunately, based on the potential form of equation (1), it is possible to find a transformation that eliminates the axial dependence, and then to use the projection.

In general, two types of trajectories can be observed. The first are trapped trajectories, which appear either periodic, or quasi periodic, see the examples in Figures $1 \mathrm{~b}$ and $1 \mathrm{~d}$. The others are non-trapped trajectories, which either follow a potential separatrix (see Figure 1c), or resemble random-walk trajectories (see Figure 1a.

The effect of the parameters introduced in section 3 can be summed up on the basis of the trajectory plots. First, the trajectories of nucleon-mass particles are trapped much more often than electron trajectories; other factors being constant. Similarly, the increase in the potential amplitude results in both the light and heavy particles being trapped more frequently. Last, no effect based on the change of RMP amplitude could be observed.

\subsection{Surfaces of SeCtion}

Surfaces of section (see Figure 2 were obtained using the procedures described above. As expected, surfaces of section change with increasing amplitude of the electrostatic perturbation $U_{0}$. The increase also leads to (2-D) confinement of individual trajectories. This is demonstrated in the surfaces of section for lithium see Figure 2 The lower threshold of the effect of electrostatic perturbation on lithium is at $0.1-0.5 \mathrm{~V}$, and this matches well with the results obtained for carbon ions and protons. The fraction of confined lithium ions is 0.81 at $20 \mathrm{~V}$. We plan to perform a 


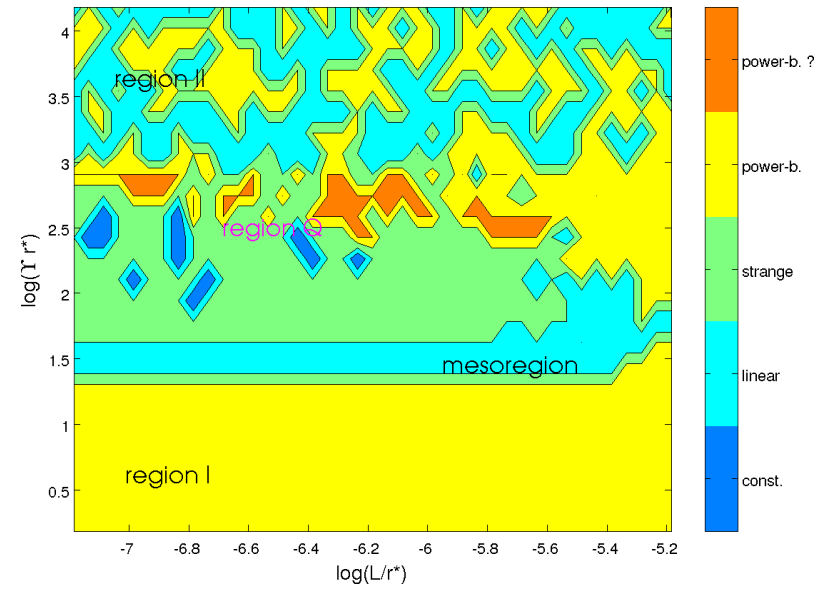

(a) ions

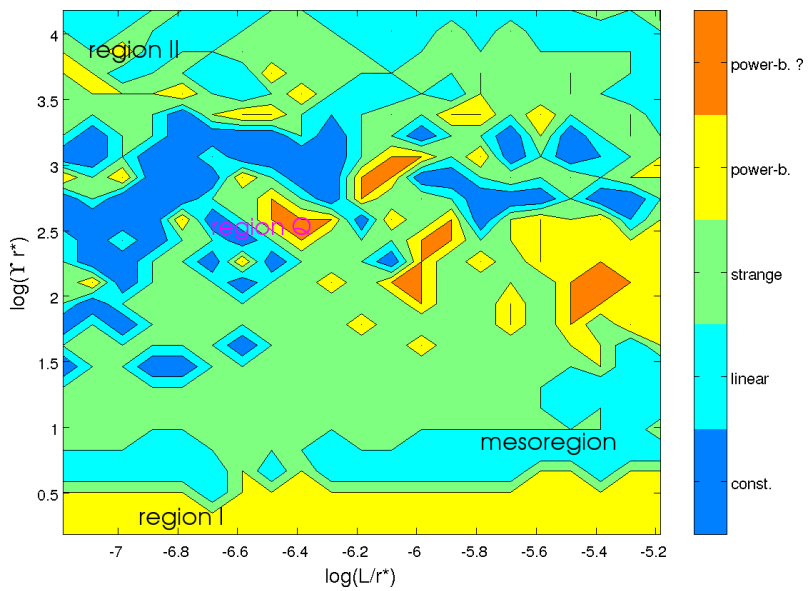

(b) protons

FiguRE 4. Figure showing different types of diffusion as a function of $\Upsilon r^{*}$ and $L / r^{*}$ for carbon ions (Fig. 4a), and protons (Fig. 4b). The different colours indicate the type of function which best approximates the time evolution of the average square radial deviation, and thus essentially the type of diffusion in the system.

similar analysis for electrons in future and - based on a comparison of the trajectories - we expect the amplitude threshold to be higher.

The investigation of the effect of $L$ on the system is inconclusive. There is no measurable difference at high amplitudes of $U_{0}$, which confirms our assumption that the ergodic layer plays very little role at such amplitudes. However, in the onset range 0.1-0.5 V, there appear to be marginally more confined trajectories in the absence of the ergodic layer. This conjecture could be further investigated in future.

\subsection{Phase space Plots}

Based on our simulations, we observe that both the increase in the amplitude of the electrostatic potential, represented by $\Upsilon$, and the increase in the amplitude of the ergodic layer $L$ in general tend to increase the diffusion, see Figure 3. This is the case for both the carbon ions (Fig. 3a) and the protons (Fig. 3b). We also studied the diffusion type based on the same parameters (see Fig. 4). It is remarkable to observe that the regions of dominant diffusion types match the regions of a certain diffusion rate, cf. Figures $4 \mathrm{a}$ and $4 \mathrm{~b}$

Our simulations also suggest that the diffusion of heavy particles follows a scaling pattern, i.e. the positions of the distinct regions remain approximately constant for particles of different mass, see Figure 3 The mappings of the diffusion types also show distinct similarities: it is possible to see transitions from one diffusion regime into another in the same span of $L / r^{*}, \Upsilon r^{*}$ for particles of different mass, see Figure 4

Unfortunately, we were unable to complete the procedure for electrons due to the enormous time requirements. We have seen, however, that the diffusion of test particles of muon mass is considerably different from the diffusion of ions. This remains to be investigated in more detail in future.

\section{Summary}

In our paper, we have attempted to ascertain whether it is electrostatic perturbation or the RMP layer that determines the particle dynamics. Based on the results presented above, we can conclude that for particles of nucleon mass at total energy $20 \mathrm{eV}$, electrostatic perturbation is the most influential factor in the system. Even at relatively low amplitudes of $0.1-0.5 \mathrm{~V}$ electrostatic perturbation affects the dynamics, and obviously dominates it at $5 \mathrm{~V}$. The increase of the amplitude of the potential results in two main effects. First, the trajectories of the particles become trapped in the potential structures. Second, the radial diffusion increases globally, which suggests that non-trapped trajectories are diffusing much more in the radial direction.

We also scanned the parametric space of the amplitudes of the electric and perturbed magnetic field in the system and calculated the radial diffusion coefficient for heavy particles. We discovered that for a particular choice of parameters $L / r^{*}, U_{0} q r^{*} / E$ the coefficient scales well with particle mass. We also observed transitions between regimes dominated by the first or second factor. In addition, we were able to study the diffusion type and its dependence on the parameters of the system, where we saw that changes in the rate of diffusion and its type are closely related.

Last, we were able to compare the effect of electrostatic perturbation and the RMP layer on electrons and heavier particles. Based on a study of their trajectories, we observed that electrostatic perturbation affects electrons as well, but its effect appears smaller than it is for heavy particles. The range of parameters where electrostatic perturbation dominates the particle dynamics is different for electrons. This suggests that the electron dynamics in the system is also different. We are planning to study electron dynamics in more detail in future. Eventually, our results should be checked by employing a more realistic form 
of the electrostatic potential, such as the HasegawaWakatani model.

\section{ACKNOWLEDGEMEnTS}

This work was supported by the Grant Agency of the Academy of Sciences of the Czech Republic No. IAA100430502 and by the Grant Agency of the Czech Republic No. GA202/07/004, and partly supported by MSMT Project No.LM2011021.

We would like to express our thanks to J. Seidl and R. Papřok for their opinions and suggestions, which they shared with us freely.

\section{REFERENCES}

[1] Evans T. E., et al. Nature Physics 2 (2006), 419-423

[2] Naulin V., Nielsen A. H., Rasmussen J. J. Phys. Plasmas, 6 (1999) 4575.

[3] Krlín L., Stöckel J., Svoboda V. Plasma Physics and Controlled Fusions, 41 (1999) 339.

[4] Krlín L., Papřok R., Svoboda V. European Physical Journal D, Vol. 48 (2008), 95-109

[5] Bleuel J. et al. New Journal of Physics, Vol. 438 (2002)

[6] Geisel T. in Lectures Notes in Physics Vol. 450, (Editors M.F. Shlesinger, G.M. Zaslavsky, U. Frisch) Springer (1995), 151-173

[7] Cahyna P., Krlín L. Czech. J. Phys. Vol. 56 (2006) 367

[8] Krlín L., Cahyna P. Czech. J. Phys. Vol. 56 (2006) Suppl. B B111. 